changes in survival rate or cell proliferation. However, it clearly promoted the induction of cell hypertrophy.

Conclusion In summary, these preliminary data suggest that SIK2 might modulate the hypertrophic response of cardiac tissue during pathological insults.

Conflict of Interest No

\section{BS25 TAM RECEPTOR AXL LOSS REGULATES SMOOTH MUSCLE CELL DIFFERENTIATION AND ACCELERATES ATHEROSCLEROSIS IN MICE}

'Lucy Mcshane, 'Danila Gurgone, ${ }^{1}$ Abdullatif Mohammed Bin-Khunayn, ${ }^{2}$ Eva Crespo, ${ }^{1}$ Gianluca Grassia, ${ }^{1}$ Neil MacRitchie, ${ }^{3}$ Mauro Giacca, ${ }^{4}$ Lorena Zentilin, ${ }^{5}$ Glykeria Karadimou, ${ }^{5}$ Gabrielle Paulsson-Berne, ${ }^{5}$ Peder S Olofsson, ${ }^{1}$ Thomas D Otto, ${ }^{2}$ Tomasz I Guzik, ${ }^{1}$ Mariola Kurowska-Stolarska, 'Pasquale Maffia. 'Centre for Immunobiology, Institute of Infection, Immunity and Inflammation, University of Glasgow, Glasgow, UK; ${ }^{2}$ Institute of Cardiovascular and Medical Sciences, University of Glasgow; ${ }^{3}$ School of Cardiovascular Medicine \& Sciences, King's College London British Heart Foundation Centre; ${ }^{4}$ Molecular Medicine Laboratory, International Centre for Genetic Engineering and Biotechnology (ICGEB): ${ }^{5}$ Center for Molecular Medicine, Karolinska Institutet

\subsection{6/heartjnl-2021-BCS.223}

Introduction The TAM receptors (Tyro3, Axl, and MerTK) are a distinct family of three receptor tyrosine kinases, namely Tyro3, Axl and MerTK, which play critical roles in cancer, inflammatory disorders and cardiovascular diseases. Axl, in particular, has been shown to influence multiple aspects of cardiovascular pathology via diverse effects on cells of both the vasculature and immune system through regulation of vascular remodelling, efferocytosis and inflammation. Clinical studies have shown that Axl is detectable in atherosclerotic plaques; however, the causal relationship between Axl and atherosclerosis is still uncertain, and results from mouse models fell short of defining the specific role(s) of Axl in the disease process.

Methods In order to quantify Axl expression in carotid endarterectomy atherosclerotic plaques we examined data from the Biobank of Karolinska Endarterectomy (BiKE). Using single-cell RNA sequencing (scRNA-seq) data from published atherosclerosis datasets we determined which cell types express Axl during pathology. Finally, we utilised an inducible atherosclerosis model in order to assess atherosclerosis formation in global Axl-deficient mice (Axl-/-). Female C57BL/6NQ (WT; $n=15)$ and Axl-/- $(n=21)$ mice were injected with $3 \times 1011$ vector genomes of AAV8-proprotein convertase subtilisin/kexin type 9 (PCSK9) and placed on a Western Diet (WD) for 12 weeks. Plaque size and percentage of necrotic core were determined in the aortic sinus using Oil Red O (ORO). Collagen content was determined using picrosirius red and polarised light microscopy. ScRNA-seq was performed to explore differences in Axl-/- vs. WT aortas at the cellular and molecular level.

Results We found expression of Axl in human carotid plaque to be significantly reduced in comparison to healthy control tissue $(\mathrm{P}=1.96 \mathrm{e}-06)$ in the BiKE cohort. Similarly, we detected less Axl RNA expression in the aortas of WDfed apolipoprotein-E-/- mice compared to WT $(\mathrm{P}<0.05)$. Analysis of published scRNA-seq databases found that Axl is expressed predominantly in the vascular smooth muscle cell (VSMC) compartment of the aortas in both healthy and atherosclerotic mice, with expression also observed in fibroblasts and macrophages. Global Axl-deficiency increased lesion size in the aortic sinus $(\mathrm{P}<0.001)$. While collagen content and necrotic core were not affected. ScRNA-seq on the aortas showed a switch versus a less contractile smooth muscle cell phenotype in Axl-/- mice compared to WT.

Conclusions In conclusion, our results indicate a protective role for Axl in atherosclerosis. The TAM receptor is reduced in diseased vessel compared to healthy controls in both human and mouse. Furthermore, global knock-out resulted in significantly increased plaque burden in mice. The necrotic core was not found to be influenced by Axl, suggesting that TAM receptor-mediated efferocytosis is not a key contributor to the role of Axl in atherosclerosis. Axl was found to be predominantly expressed in the VSMC compartment in the aortas of both healthy and diseased mice. Furthermore, Axl deficiency promoted VSMC phenotypic switching. These data support the hypothesis of a beneficial role of Axl in atherosclerosis via modulation of smooth muscle cell phenotype.

Conflict of Interest none

\section{BS26 THE PARTNERSHIPS IN CONGENITAL HEART DISEASE IN AFRICA STUDY (PROTEA): CLINICAL CHARACTERISTICS AND GENETIC FINDINGS FROM A SOUTH AFRICAN CONGENITAL HEART DISEASE COHORT}

${ }^{1}$ Timothy Spracklen, ${ }^{1}$ Thomas Aldersley, ${ }^{1}$ Nicole Saacks, ${ }^{1}$ Bianca de Koning ${ }^{1} J o h n$ Lawrenson, 'Paul Human, ${ }^{2}$ James Eales, ${ }^{1}$ Blanche Cupido, 'George Comitis, ${ }^{1}$ Rik De Decker, ${ }^{3}$ Barend Fourie, 'Lenise Swanson, ${ }^{1}$ Alexia Joachim, ${ }^{1}$ Phaphama Magadla, ${ }^{1}$ Malebogo Ngoepe, ${ }^{1}$ Liam Swanson, ${ }^{2}$ Alistair Revell, ${ }^{1}$ Raj Ramesar, ${ }^{1}$ Gasnat Shaboodien, ${ }^{1}$ Andre Brooks, ${ }^{1}$ Karen Sliwa, ${ }^{1} J o h n$ Anthony, ${ }^{1}$ Ayesha Osman, ${ }^{2}$ Bernard Keavney, 'Liesl Zuhlke. 'University of Cape Town, Cape Town, South Africa; ${ }^{2}$ University of Manchester; ${ }^{3}$ University of Stellenbosch

\subsection{6/heartjnl-2021-BCS.224}

Introduction Congenital heart disease (CHD) is the most common birth defect and a significant cause of paediatric morbidity and mortality worldwide. Epidemiological data from Africa are lacking, although this information is of importance in determining the burden of CHD and guiding policy. As a multifactorial disease, the role of genetic factors in $\mathrm{CHD}$ is increasingly recognised. However, the genetic contribution to CHD remains relatively unexplored in Africa. The Partnerships in CHD in Africa (PROTEA) project was established to better understand the epidemiology and genetics of CHD in subSaharan Africa. The aim of this investigation is to describe the clinical and genetic characteristics of a cohort of CHD patients from the Western Cape, South Africa.

Methods PROTEA is a multicentre, prospective registry of CHD patients, recruited from seven hospitals in the Western Cape, South Africa. Patients with any structural CHD were eligible for inclusion, this excluded patients with isolated patent foramen ovale, peripheral pulmonary stenosis or patent ductus arteriosus in premature infants. Some of these patients were consented into the genetics study, for which a DNA biorepository was established. These patients were investigated using exome sequencing and/or chromosomal microarray (CMA) to identify disease-causing mutations or copy number variants in established CHD genes.

Results A total of 1,473 participants were recruited into the PROTEA registry between April 2017 and March 2019 (median age 1.9 years, 51\% male). Compared to international cohorts, ventricular (PR: 1.8, 95\%CI: 1.63-1.97) and atrial (PR: 1.4, 95\%CI: 1.20-1.57) septal defects were significantly 